\title{
STRUCTURES DÉMOGRAPHIQUES DE POPULATIONS ADULTES DE GRENOUILLES VERTES DU COMPLEXE ESCULENTA
}

\author{
par A. NEVEU \\ Laboratoire d'Écologie Hydrobiologique - INRA \\ 65, rue de St Brieuc - 35042 RENNES CEDEX
}

Reçu le 24 décembre 1990

Accepté le 27 mai 1991

Received 24 December, 1990

Accepted 27 May, 1991

\section{RÉSUMÉ}

Cette étude concerne 763 Rana lessonae et 306 Rana esculenta capturées au cours de 5 années dans différents sites des Pays de Vilaine, dans la partie ouest de la France. Les biotopes prospectés, étangs, mares, marais, présentent des structures spécifiques différentes. Les grenouilles ont été mesurées et sexées, leur âge a été déterminé à partir de coupes dans la diaphyse des tibias. Les échantillons sont proportionnels à la densité réelle des populations en place, contrairement à la majorité des études.

Les histogrammes des fréquences des longueurs font apparaître deux groupes d'animaux : les subadultes et les adultes. Les premiers sont âgés de 1 an, les seconds de 2 et 3 ans, quelques individus dépassent 4 ans. Si la croissance est meilleure chez les mâles la première année, c'est l'inverse par la suite. La croissance de $R$. esculenta est supérieure à celle de $R$. lessonae, ce qui donne des tailles maximales plus fortes.

La croissance de $R$. esculenta est variable suivant les sites. Elle est meilleure dans les zones agricoles où les eaux sont plus eutrophisées, mais les densités de populations sont aussi plus faibles. La croissance est variable suivant les années : jusqu'en 1982 les tailles moyennes sont supérieures à celles des années suivantes.

Le sex ratio est généralement proche de 1 , sauf pour les populations de $R$. esculenta triploïdes constituées en majorité de mâles.

Le taux annuel de mortalité augmente avec l'âge, seulement $7 \%$ des $R$. lessonae et $4 \%$ des $R$. esculenta atteignent l'âge de 4 ans.

\section{DEMOGRAPHIC STRUCTURES OF GREEN FROGS ADULT POPULATIONS OF THE ESCULENTA COMPLEX}

\section{SUMMARY}

A total of 763 pool frogs (Rana lessonae) and 306 edible frogs (Rana esculenta) were collected at different sites in the "Pays de Vilaine", in western part of France, during five years. Each type of biotops ponds, pools, marshes, has a particular species structure. All frogs were measured, sexed and aged by analysis of tibia diaphysis sections. Each sample was proportionnal to densities of standing populations contrary to other studies.

Lengths frequencies distributions show two groups of frogs : subadults and adults. Subadults are one year old, adults mainly two or three years old, frogs more four years old are scarce. Growth in males is better than in females only during the first year. The growth rate and the maximal size in Rana esculenta is higher than in Rana lessonae.

$R$. esculenta growth is related to sample site. Growth is faster in agricultural areas where waters are more eutrophicated and frogs populations densities smaller. Growth changes according to year and until 1982 mean lengths were greater than following years. 
Sex ratio is generally very close to one, except triploïd $R$. esculenta populations composed mainly by males.

The annual rate of mortality increases with age, only 7 per cent $R$. lessonae and 4 per cent $R$. esculenta adults reach 4 years old.

\section{INTRODUCTION}

S'il existe de nombreux travaux sur la démographie de certains groupes zoologiques tels que les poissons, très peu concernent les batraciens et encore moins les grenouilles européennes. Cet état de fait s'explique par l'absence de structures permettant une lecture facile de l'âge et par certaines difficultés de récolte des divers stades. Quelques études récentes s'intéressent à la détermination de l'âge chez la grenouille rousse $R$. temporaria (GIBBONS et Mc CARTHY, 1983 ; GUYETANT et al., 1984 ; GUYETANT et al., 1988 ; RYSER, 1988) ou encore chez le crapaud commun Bufo bufo (HEMELAAR et VAN GELDER, 1980 ; GITTINS et al., 1982). Les données concernant le complexe esculenta sont limitées à celles de BORKIN et al., (1979), FRANCILLON et CASTANET (1985). Dans la plupart des cas il s'agit d'études ponctuelles, surtout méthodologiques, limitées à des sondages sur des individus matures lors des rassemblements de reproduction.

Depuis plusieurs années les populations de grenouilles vertes de l'ouest de la France, entre Rennes et Redon, sont suivies régulièrement. Les premiers résultats sur les structures spécifiques montrent que les peuplements sont constitués par un mélange de Rana lessonae CAMERANO et de Rana esculenta $L$., cette dernière pouvant présenter un certain nombre de triploïdes (REGNIER et NEVEU, 1986). Une première approche démographique fut réalisée entre 1981-1982 essentiellement à partir des distributions en classes de tailles complétées par quelques mesures squeletto-chronologiques (REGNIER, 1983).

Les résultats actuels concernent une série d'échantillons prélevée de 1981 à 1985 dans divers biotopes. Ils peuvent servir de base à une analyse de l'âge individuel à partir de la composition normale des populations, c'est-à-dire chaque âge étant représenté proportionnellement à sa densité réelle estimée par capture-marquage-recapture (données non publiées).

\section{MATÉRIEL ET MÉTHODES}

Les grenouilles ont été capturées soit à la ligne de jour, soit à la main de nuit. La comparaison des deux techniques ne révèle pas de différence significative quant à une sélectivité vis-à-vis du sexe, de l'espèce ou de la taille. Ceci étant confirmé par les résultats obtenus à partir d'animaux marqués (données non publiées). Dans certaines circonstances, en particulier par manque d'entraînement du pêcheur, les captures à la ligne peuvent prendre un peu moins de petits individus (entre 30 et $50 \mathrm{~mm}$ ).

Les animaux captifs sont anesthésiés au chloroforme puis stockés dans l'alcool à $95^{\circ}$. Au laboratoire, le sexe est déterminé sur les caractères externes ou par dissection. Les animaux classés comme "immatures" ne possèdent pas de caractères sexuels externes visibles. La longueur totale (museau-orifice cloacal) est mesurée avec une règle ou un compas à pointes sèches. L'espèce est déterminée à partir des critères biométriques définis par REGNIER et NEVEU (1986). Pour l'analyse ostéologique, les os des tibias (en fait les tibio-fibulaires) sont prélevés sur chaque individu, nettoyés et décalcifiés à l'acide nitrique à $5 \%$ pendant 10 à 12 heures. Un segment central de la diaphyse, au-dessus du trou nourricier, est prélevé pour analyse histologique. Les coupes de 20-30 $\mu \mathrm{m}$ réalisées au cryomicrotome, sont ensuite colorées à l'hématoxyline de Ehrlich pendant 10 à 15 minutes et montées entre lame et lamelle à l'Aquamount. Les observations sont effectuées au microscope à un grossissement de 60 fois et complétées par un dessin des contours des lignes d'arrêt de croissance (LAC) grâce à un tube à dessin. Ne sont considérées comme LAC que les lignes bien nettes faisant le tour complet de l'os ; certaines fausses marques peuvent être visibles, mais elles sont plus diffuses et incomplètes. 
La période d'échantillonnage étant limitée à l'été, les jeunes grenouilles de l'année, facilement repérables, sont éliminées de l'étude.

Les sites prospectés ont été choisis pour représenter les principaux biotopes de la région à savoir (fig. 1) :

- les étangs : surfaces supérieures à $1.000 \mathrm{~m}^{2}$, à niveau d'eau stable, vidangeables la plupart du temps (sites 1 et 5 ).

- les mares : surfaces réduites à quelques dizaines de $\mathrm{m}^{2}$, à niveau d'eau assez stable, souvent recouvertes de végétaux (site 4).

- les marais : surfaces variables, avec des zones d'eaux permanentes riches en végétaux hydrophytes (sites 2 et 3 ).

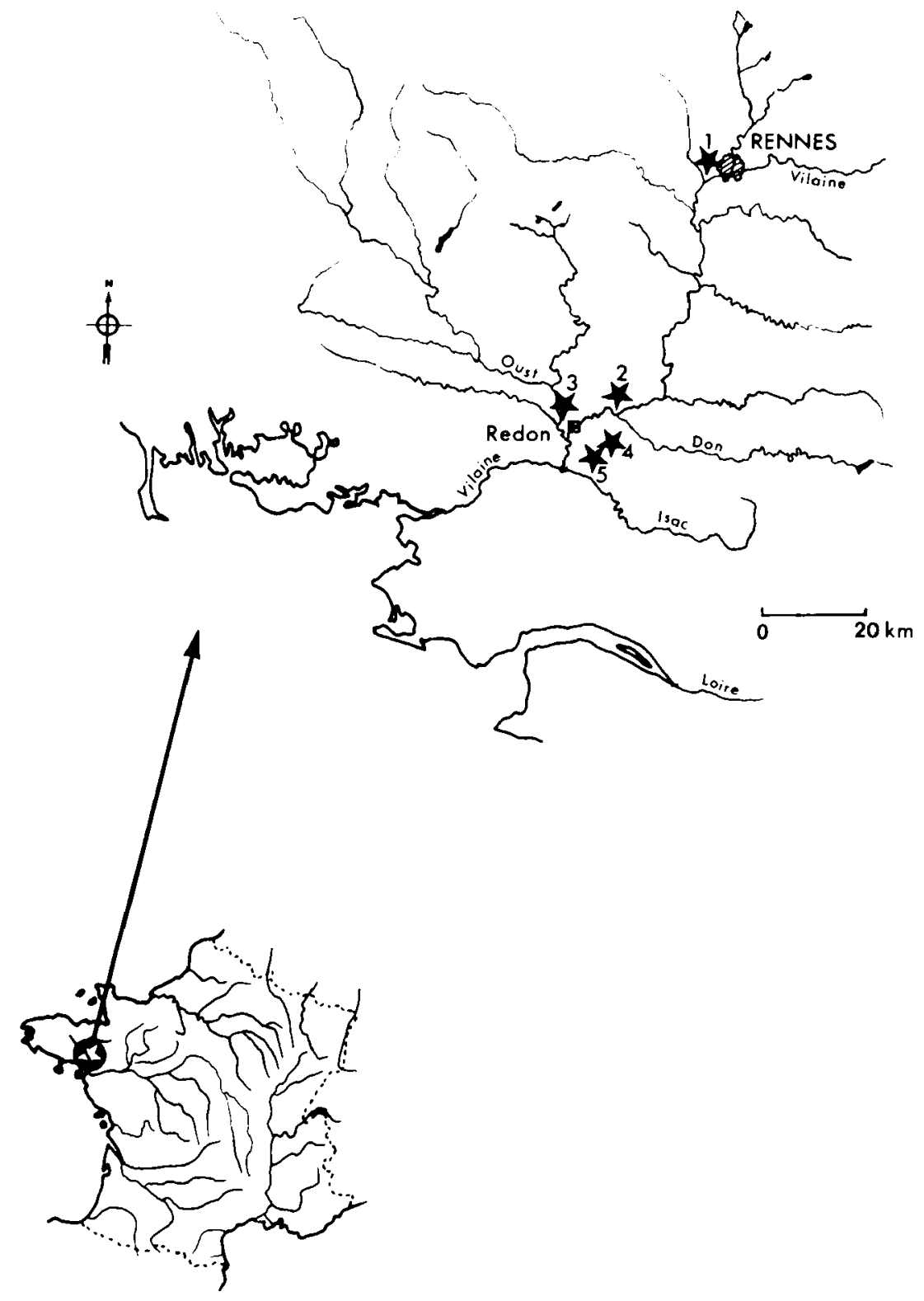

Figure 1 : Localisation des sites d'étude.

Figure 1 : Location of collecting sites. 
Les populations de grenouilles vertes des sites 1 et 5 sont composées de $R$. esculenta avec quelques $R$. lessonae, celles des sites 2 et 3 sont en majorité des $R$. lessonae. Le site 4 est colonisé par des $R$. esculenta triploïdes avec une très forte proportion de mâles (REGNIER et NEVEU, 1986).

Seuls les échantillons les plus importants ont été analysés, l'ensemble représentant $763 R$. lessonae et $306 R$. esculenta.

Pour le traitement des résultats, la nomenclature utilisée classiquement sur les poissons est adoptée : $1+$ concerne une grenouille capturée au cours de l'été suivant l'année de sa naissance, 2+ concerne une capture le $2^{e}$ été, etc... Les tests statistiques courants sont utilisés pour comparer les croissances : test $Z$ de la différence des moyennes pour un nombre d'individus supérieur à 30, test $t$ de Student pour un nombre inférieur à 30 et test $F$ de Fisher pour la variance. Dans ce travail, on supposera que les distributions des tailles ne s'éloignent pas trop d'une loi normale, le test t étant particulièrement robuste à ce sujet (SCHERER, 1984). Dans le cas de la comparaison des distributions des fréquences de tailles, le test de Kolmogorov-Smirnov est employé.

\section{RÉSULTATS}

Les structures démographiques peuvent être analysées suivant deux modes soit par l'étude des histogrammes des longueurs, soit par la distribution des classes d'âge à partir de critères d'âge détectables directement.

Cette détermination des différentes cohortes annuelles permet ensuite une approche des taux de mortalité et des vitesses de croissance en fonction du sexe, du site, etc...

\section{LES STRUCTURES DÉMOGRAPHIQUES}

\subsection{Structures en classes de longueur}

Le but est d'essayer de faire apparaître des cohortes homogènes dans leur croissance correspondant si possible à des âges.

\subsection{1. Évolution estivale}

Compte tenu d'éventuelles différences, il est nécessaire de traiter indépendamment $R$. lessonae et $R$. esculenta, mais aussi les mâles et les femelles, de même que les sites. Le marais du site 3 peut servir de référence pour $R$. lessonae et l'étang du site 5 pour R. esculenta.

Sur le site $3, R$. lessonae montre une évolution estivale dans les tailles de certains individus (fig. 2A). La fourchette des tailles est réduite : 30 à $65 \mathrm{~mm}$. En juillet deux catégories apparaissent : $30-46 \mathrm{~mm}$ et $47-62 \mathrm{~mm}$, les animaux du premier groupe ne présentant pas de caractères sexuels secondaires externes (sacs vocaux des mâles), ils peuvent être considérés comme des immatures de l'année précédente, les juvéniles de l'année n'étant pas encore métamorphosés. Le $2^{e}$ groupe est constitué d'adultes sexables. Au cours de l'été, le $1^{\text {er }}$ groupe s'incorpore progressivement au $2^{e}$. En septembre la population a pratiquement un aspect unimodal. Les mâles et les femelles suivent une évolution très proche ; les individus les plus grands sont des femelles. Les caractères sexuels secondaires apparaissent à 44-46 $\mathrm{mm}$ pour la plupart des mâles.

Pour $R$. esculenta sur le site 5 l'aspect des histogrammes est différent (fig. 2B). Les tailles sont plus variables de 32 à $94 \mathrm{~mm}$ chez les femelles et de 32 à $72 \mathrm{~mm}$ chez les mâles. En juillet, les immatures mesurent de 32 à $52 \mathrm{~mm}$, ils s'incorporent progressivement au reste de la population au cours de l'été. En septembre, la population mâle est bimodale, alors que les femelles sont plus hétérogènes. Les caractères sexuels secondaires externes apparaissent entre 48 et $52 \mathrm{~mm}$, à une taille légèrement supérieure à celle observée chez R. lessonae.

En septembre, tous les mâles possèdent leurs caractères sexuels secondaires, les structures en classes de tailles sont plus homogènes, la croissance s'arrête avant l'hivernage en octobre. Cette époque est donc plus propice pour les analyses démographiques. 
A
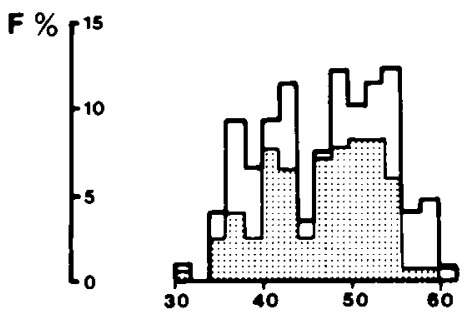

JUILLET

$\prod q$

$\int_{0}^{15}$

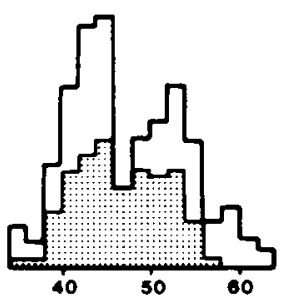

\% $\sigma$

AOUT

$\int_{-10}^{20}$

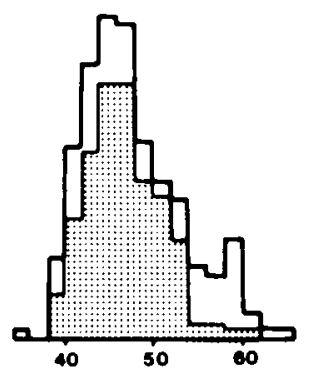

SEPTEMBRE

2

$\int_{0}$

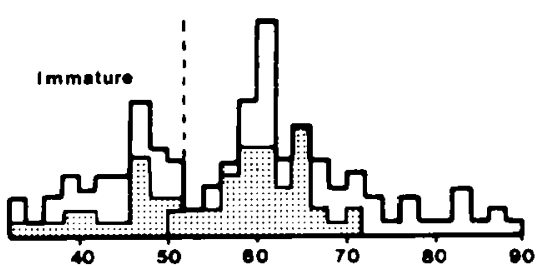

JUILLET

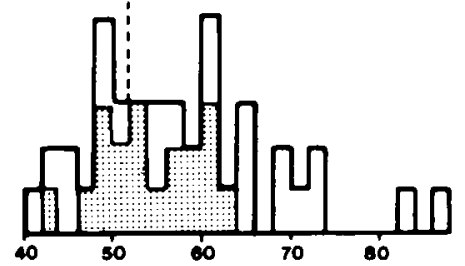

AOUT

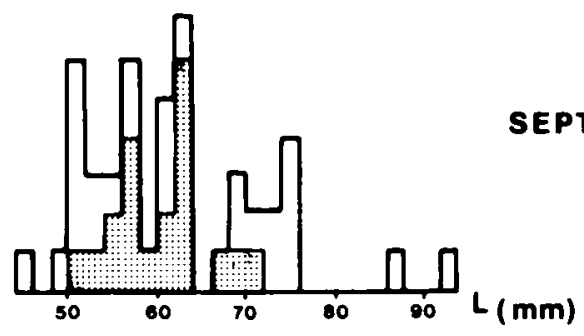

Figure 2 : Evolution des structures démographiques au cours de l'été 1982 (A. Rana lessonæ, site 3 - B. Rana esculenta, site 5).

Figure 2 : Length frequency distributions of green frogs during summer 1982 (A. Rana lessonæ, site 3 - B. Rana esculenta, site 5). 


\subsubsection{Variations interannuelles}

Pour $R$. lessonae, sur le site 3 l'aspect général en septembre ne change guère au cours de trois années (fig. 3A). En effet l'application du test de Kolmogorov-Smirnov n'est significatif au seuil de $5 \%$ que dans le cas des femelles de 1985, mais une translation de l'histogramme de 3 classes de longueur vers les grandes tailles ne montre plus de différence. Autrement dit, la forme générale est bien constante d'une année à l'autre. Les femelles présentent toujours deux grands types d'individus: les subadultes de l'année précédente et tous les autres; les mâles sont plus homogènes avec des différences intergroupes moins fortes (test de Kolmogorov non significatif, à la limite pour les mâles de 1985, dans ce cas une translation des histogrammes vers les grandes tailles rend le test négatif, il n'y a donc pas de différence dans la forme).

Pour $R$. esculenta la comparaison de quatre années de prélèvement sur le site 5 montre des résultats proches, mais avec une plus grande diversité des tailles (fig. 3B).

Quelle que soit l'époque de l'année, la majorité des structures démographiques à partir de la taille ne fait apparaître au mieux que deux types d'individus. II n'est pas possible d'extraire des cohortes qui correspondraient à des classes d'âge.
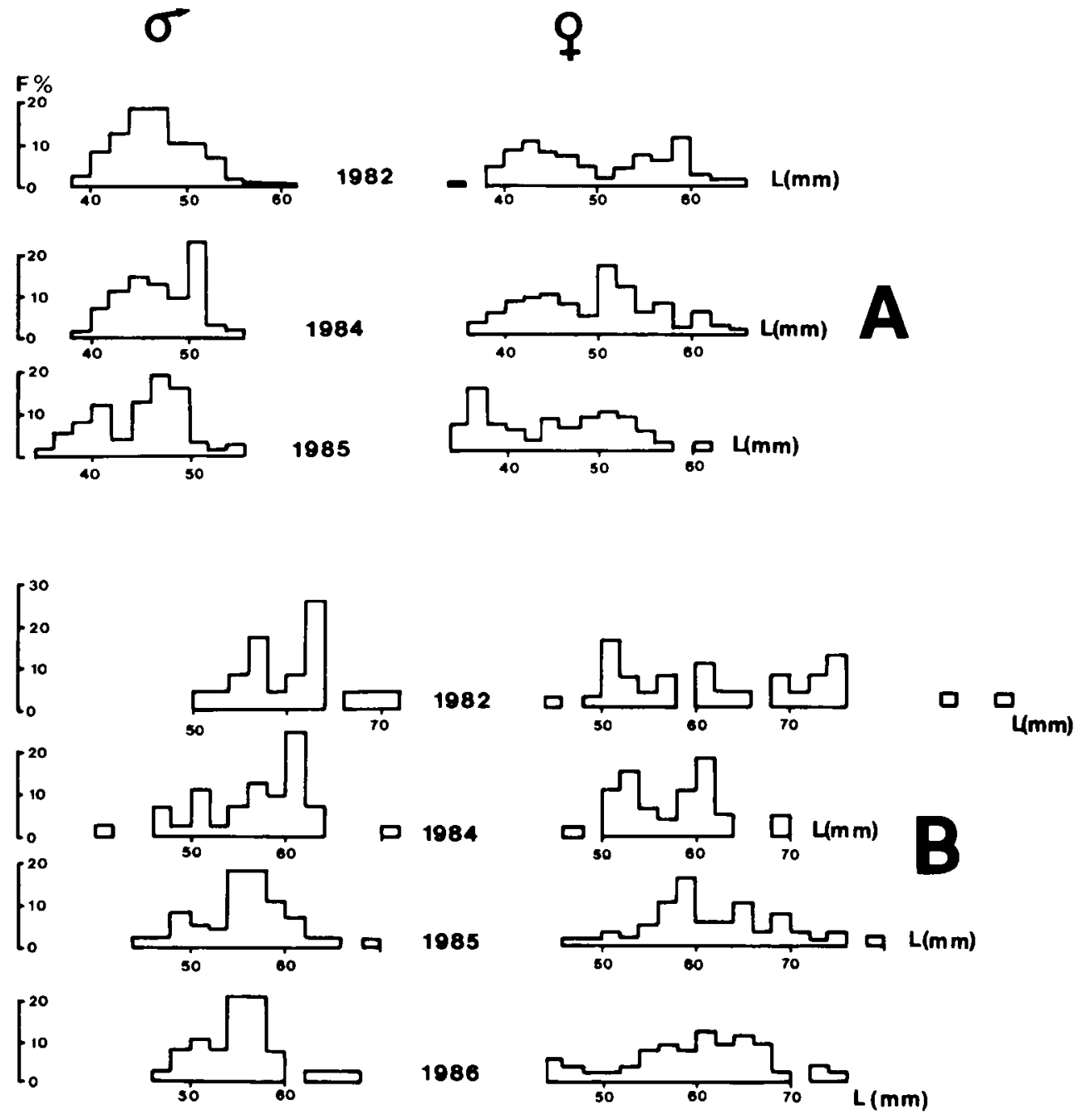

Figure 3 : Evolution des structures démographiques au cours des années (A. Rana lessonæ, site 3 - B. Rana esculenta, site 5).

Figure 3 : Length frequency distributions of green frogs through different years (A. Rana Jessonæ, site 3 - B. Rana esculenta, site 5). 


\subsection{Structures en classes d'âge}

L'analyse des coupes d'os et le dénombrement des LAC correspondant aux hivers permettent de déterminer l'âge de chaque individu.

Pour le site 3 , les $R$. lessonae sont représentés par 3 catégories dominantes d'individus âgées de 1 à 3 ans, les individus plus âgés sont très rares (fig. 4). L'hétérogénéité des tailles des plus petits animaux en juillet tend à disparaître en septembre (fig. 4 A-B).Les adultes qui représentent le $2^{\mathrm{e}}$ groupe sur la figure 3 sont constitués de 2 et 3 ans. L'aspect des structures démographiques de 1982 est comparable à celui de 1984 avec quelques individus de 4 ans. Les tailles des mâles pour un âge donné sont plus homogènes que celles des femelles, qui ont tendance à présenter une bimodalité. De même les tailles des individus d'un an sont généralement plus homogènes que celles des individus plus âgés, compte tenu de la précision des mesures.

L'aspect des structures démographiques de $R$. lessonae sur le site 2 est comparable, les intervalles des tailles pour chaque âge sont plus resserrés (fig. 4D). Dans les deux sites les individus les plus âgés sont des femelles de 5 ans.

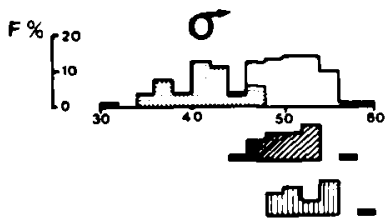

A
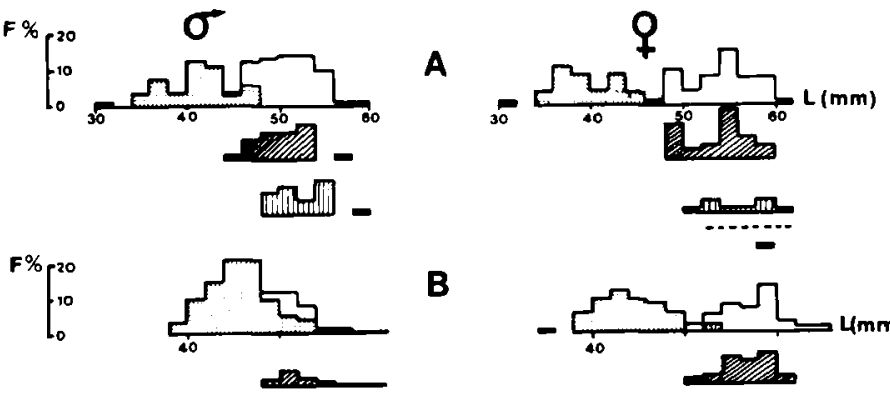

B

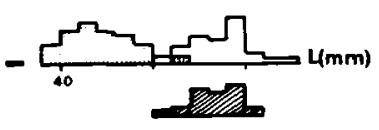

${ }^{F} \int_{20}^{30}$

min.m

田 -

C
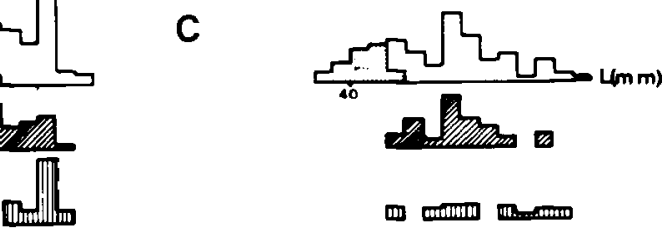

m
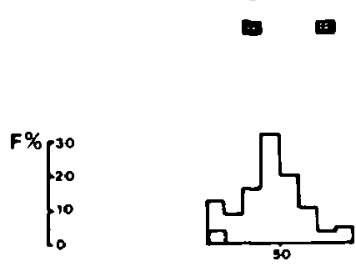

D
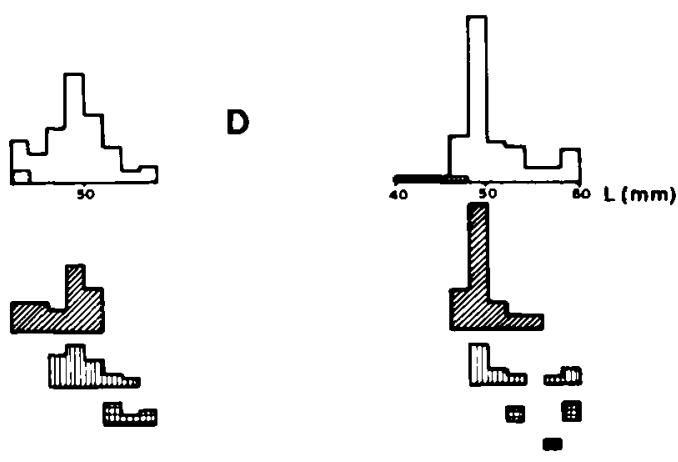

$\square 1 \square 2$

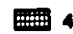

5 Ages

Figure 4 : Structure en classes d'âge de différentes populations de Rana lessonæ (A. Juillet 1982, site 3 - B. Septembre 1982, site 3 - C. Septembre 1984, site 3 - D. Septembre 1985, site 2).

Figure 4 : Age frequency distributions of Rana lessonæe populations in study area (A. July 1982, site 3 - B. September 1982, site 3 - C. September 1984, site 3 D. September 1985, site 2). 
Pour $R$. esculenta le site 5 montre des résultats semblables (fig. 5 ). Les tailles sont plus diversifiées pour chaque âge surtout chez les individus de 1 an. Les différences interannuelles sont plus nettes. Le plus vieil individu est une femelle de 6 ans.

Certains sites peuvent être fréquentés par plus d'individus de 1 an comme le site 1 (fig. 5D). Autre particularité : le cas du site 4 peuplé de triploïdes mâles de 1 à 3 ans (fig. 5E).
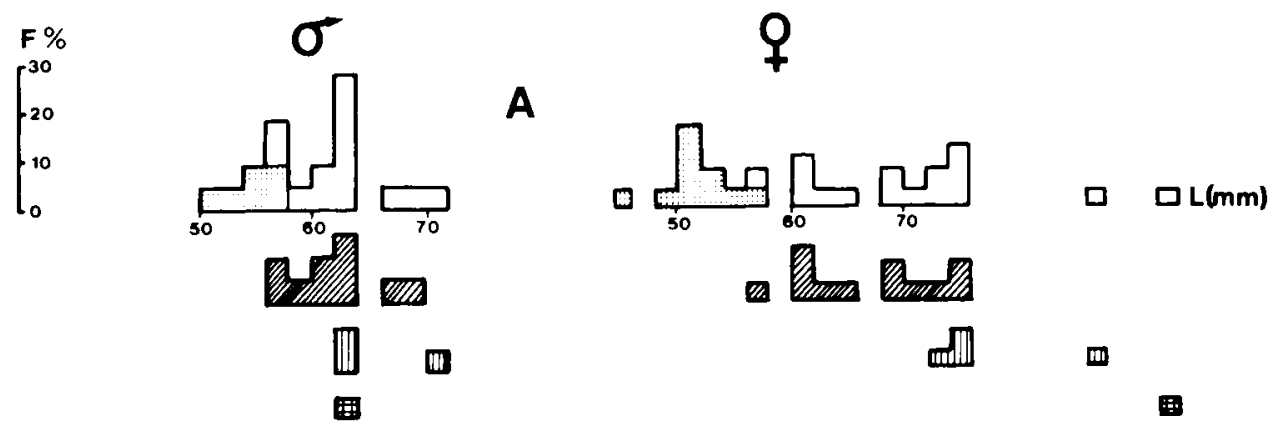

$\int_{-20}^{30}$

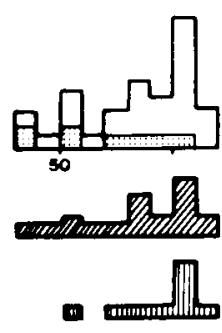

B

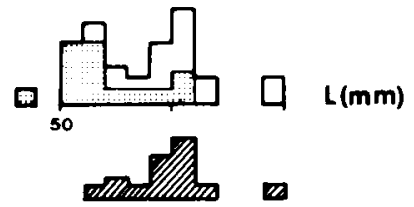

1

III III

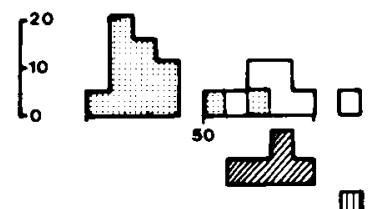

C
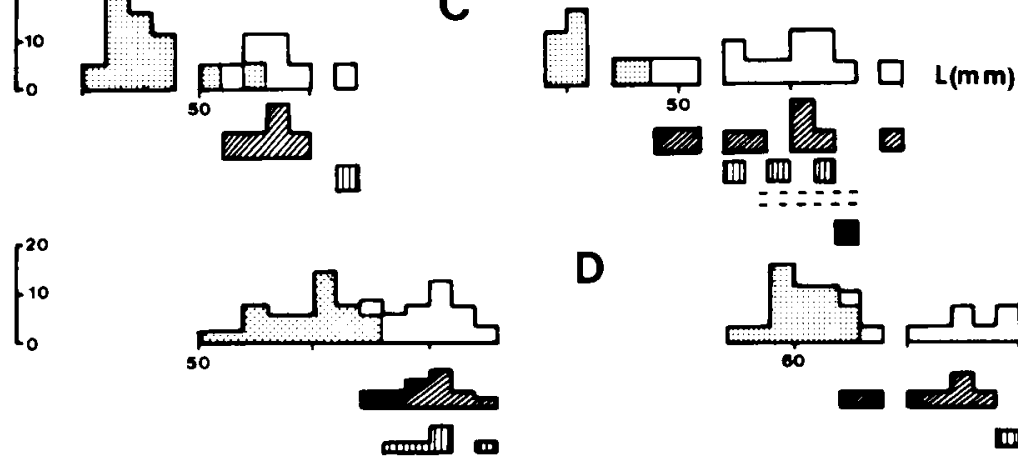

D

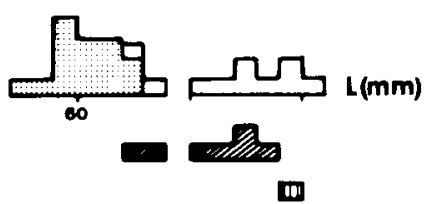

国

$\left[\begin{array}{l}40 \\ 30 \\ 20 \\ 10\end{array}\right.$

E
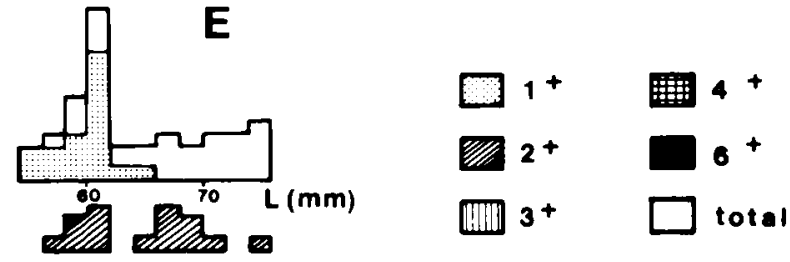

$\boldsymbol{m}$

Figure 5 : Structures en classes d'âge de différentes populations de Rana esculenta (A. Septembre 1982, site 5 - B. Septembre 1984, site 5 - C. Septembre 1984, site 3 - D. Septembre 1982, site 1 - E. Septembre 1981, site 4).

Figure 5 : Age frequency of Rana esculenta populations in study area (A. September 1982, site 3 - B. September 1984, site 5 - C. September 1984, site 3 - D. September 1982, site 1 - E. September 1981, site 4). 
Quelle que soit l'espèce, il y a donc une certaine homogénéité dans les structures démographiques d'une année ou d'un site à l'autre. Les deux catégories d'animaux trouvés à partir des classes de tailles correspondent bien pour la $1^{\mathrm{Te}}$ aux subadultes âgés de 1 an, mais pour la $2^{\mathrm{e}}$ à des adultes de 2 ans et plus.

\subsection{Rapport entre les sexes}

Le sex-ratio est variable suivant les échantillons. L'ensemble des $R$. lessonae examiné est composé par 429 mâles et 334 femelles, soit un rapport de 1,28. Pour $R$. esculentail faut distinguer les populations "normales" constituées par des diploïdes où il y a 143 mâles pour 123 femelles, soit un rapport de 1,16, des populations triploïdes du site 4 où l'échantillon de septembre 1981 était constitué uniquement de mâles. Par la suite, quelques très rares femelles ont pu être examinées sur ce site.

Au niveau des classes d'âge, si les plus grands individus sont des femelles, le sex-ratio n'augmente pas visiblement avec l'âge.

\section{IMPORTANCE RELATIVE DES DIVERS AGES - TAUX DE MORTALITÉ}

En regroupant les données régionales, il est possible d'avoir une idée des taux de mortalité (fig. 6). Chez $R$. lessonae, $21 \%$ des individus disparaissent entre 1 et 2 ans, $52 \%$ entre 2 et 3 ans et $82 \%$ entre 3 et 4 ans. Les chiffres pour $R$. esculenta sont proches avec respectivement $20 \%, 58 \%$ et $87 \%$. L'âge associé aux dépenses pour la reproduction, sont la cause principale de cette augmentation des mortalités. Seulement $7 \%$ des $R$. lessonae atteignent l'âge de 4 étés et $4 \%$ des $R$. esculenta, quel que soit leur sexe.

La part relative représentée par chaque âge varie suivant le site et l'année, même en comparant le même mois (septembre). La fréquence des $1+$ est très variable, surtout chez R. lessonae et chez les mâles, ils représentent 3,7 \% du stock pour le site 2 en 1985 contre $83,8 \%$ en 1982 pour le site 3 (fig. 6). Chez $R$. esculenta, les variations sont plus limitées. II en est de même sur les sites "étangs" (sites 1,5) par rapport aux marais (sites 2, 3). Pour l'ensemble des captures, les courbes correspondant à une tendance régionale moyenne sont régulières et assez comparables.

\section{VARIATIONS DE LA CROISSANCE}

Pour chaque âge il est possible de calculer une taille moyenne et de suivre l'influence de divers paramètres.

\subsection{Croissance moyenne régionale}

Le calcul de la taille moyenne pour l'ensemble des échantillons de septembre montre que celle-ci est différente entre $R$. lessonae et $R$. esculenta (Tab. I).

Pour les âges $1+$ à 4+, la croissance est plus lente chez $R$. lessonae. Les femelles, plus petites que les mâles la $1^{\text {re }}$ année présentent une taille supérieure par la suite. Le taux de croissance annuel $\left(\mathrm{dL}=\left(\mathrm{L}_{2}-\mathrm{L}_{1}\right) / L_{1}\right)$ et la variabilité des tailles pour un âge donné sont légèrement supérieurs chez $R$. esculenta. En particulier chez les femelles la capture de quelques grenouillettes montre que ces animaux mesurent de 20 à $25 \mathrm{~mm}$, la longueur du corps double donc pendant la première année de croissance. 


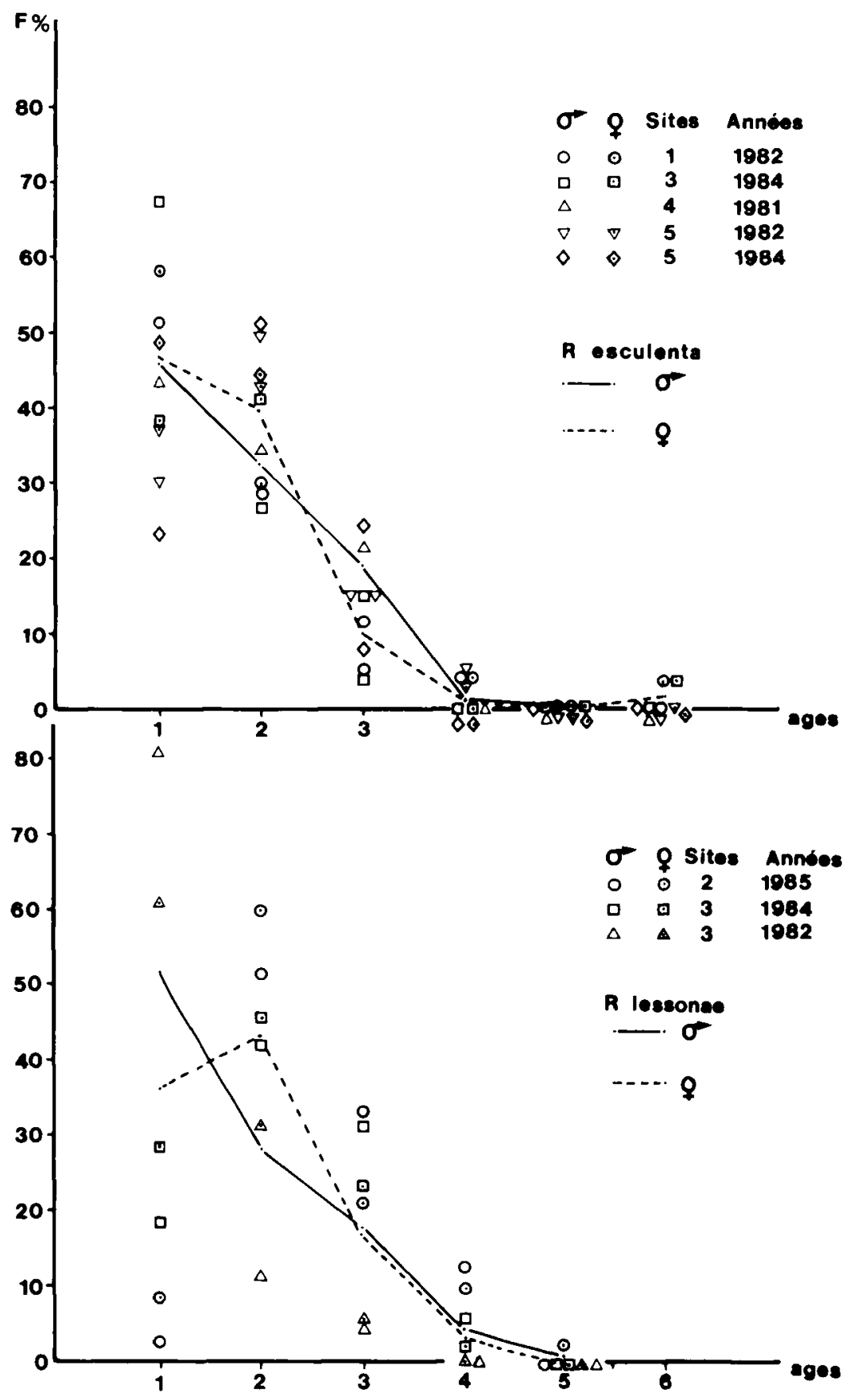

Figure 6 : Importance relative des différentes classes d'âges (courbes de la moyenne régionale pondérée).

Figure 6 : Age composition of green frogs populations in study area (curves fitting of weighted mean). 
Bull. Fr. Pêche Piscic. (1991) $321: 55-71 \quad-65-$

Tableau I : Tailles moyennes de chaque classe d'âge en septembre et taux de croissance annuel pour l'ensemble des échantillons (Moyenne régionale pondérée) ( $n=$ nombre d'individus $; m=$ longueur moyenne en $\mathrm{mm} ; \sigma=$ écart type ; $d L=$ taux de croissance annuel en pourcentage).

Table I: Mean lengths of each age in september and annual growth rate for all frogs samples combined (weighted mean for study area) ( $n=$ green frogs numbers $; m=$ mean length in $\mathrm{mm} ; \sigma=$ standard deviation $; \mathrm{dL}=$ annual growth rate pourcentage).

Ages

\begin{tabular}{llll}
\hline $1+$ & $2+$ & $3+$ & $4+$ \\
\hline
\end{tabular}

R. esculenta

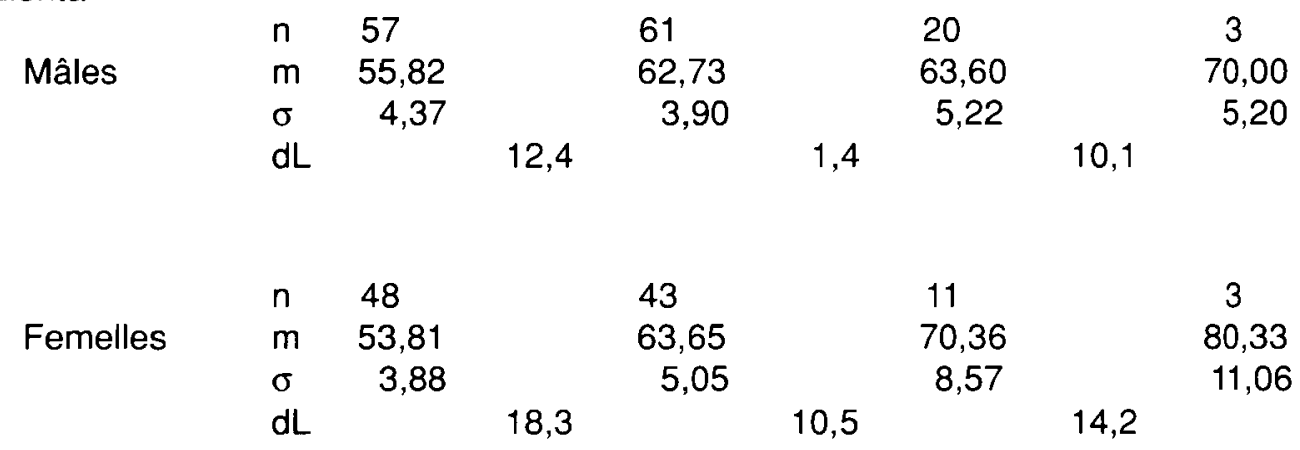

R. lessonae

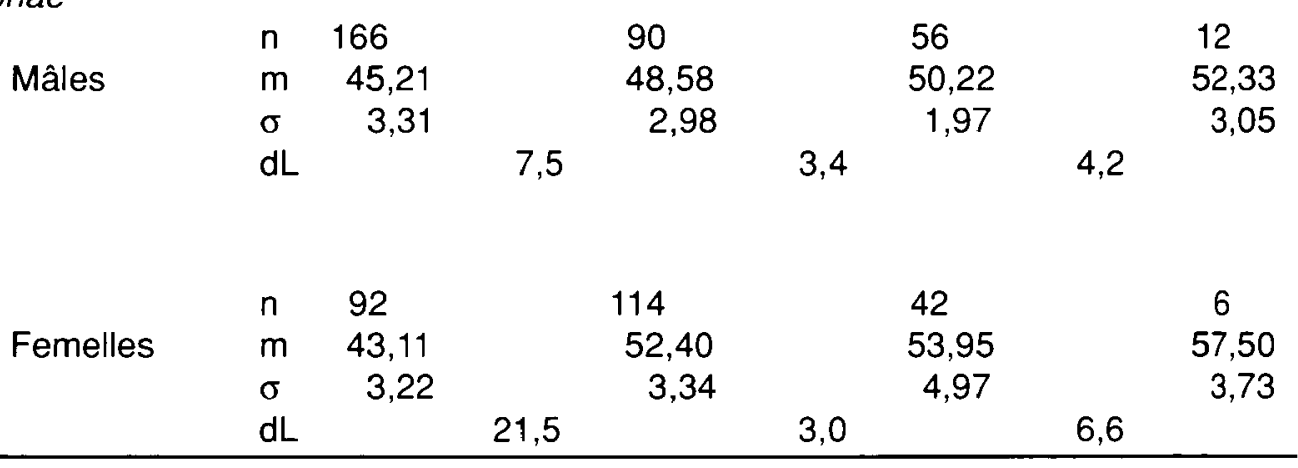

\subsection{Influence du sexe}

L'ensemble des captures montre une meilleure croissance des femelles (Tab. II et III). Généralement, la variabilité de la taille est comparable entre les deux sexes, la plupart des résultats du test $F$ sur les variances n'étant pas significative. II arrive quelquefois que celle des femelles soit plus diversifiée en particulier chez $R$. lessonae, pour une taille moyenne semblable.

La différence de taille généralement en faveur des femelles, sauf pour les $1+$, n'est pas toujours significative en particulier chez $R$. esculenta. 
Bull. Fr. Pêche Piscic. (1991) $321: 55-71 \quad-66-$

Tableau II : Tailles moyennes de chaque classe d'âge pour les principaux échantillons.

Table II : Mean lengths of green frogs for each age and sample.

\begin{tabular}{|c|c|c|c|c|c|c|c|c|c|}
\hline \multirow{3}{*}{$\begin{array}{c}\text { R. lessonae } \\
\text { Site } 2\end{array}$} & & \multicolumn{4}{|c|}{ Ages mâles } & \multicolumn{4}{|c|}{ Ages femelles } \\
\hline & & $1+$ & $2+$ & $3+$ & $4+$ & $1+$ & $2+$ & $3+$ & $4+$ \\
\hline & & & & & & & & & \\
\hline \multirow{3}{*}{$\begin{array}{c}\text { Septembre } \\
85\end{array}$} & $\mathrm{n}$ & 2 & 27 & 18 & 6 & 4 & 33 & 12 & 5 \\
\hline & $\mathrm{m}$ & 43,00 & 47,74 & 49,67 & 54,67 & 44,00 & 49,88 & 52,00 & 56,60 \\
\hline & $\sigma$ & 0,00 & 2,73 & 2,31 & 1,80 & 2,58 & 2,03 & 3,87 & 2,94 \\
\hline \multicolumn{10}{|l|}{ Site 3} \\
\hline \multirow{3}{*}{$\begin{array}{l}\text { Juillet } \\
\qquad 82\end{array}$} & $n$ & 49 & 32 & 24 & & 36 & 32 & 10 & \\
\hline & $\mathrm{m}$ & 41,00 & 50,44 & 52,57 & & 39,83 & 57,75 & 56,00 & \\
\hline & $\sigma$ & 3,73 & 2,66 & 2,64 & & 3,54 & 3,31 & 3,26 & \\
\hline \multirow{3}{*}{$\begin{array}{c}\text { Septembre } \\
82\end{array}$} & $n$ & 145 & 21 & 7 & & 57 & 31 & 6 & \\
\hline & $\mathrm{m}$ & 45,67 & 52,52 & 51,86 & & 44,05 & 56,55 & 60,67 & \\
\hline & $\sigma$ & 3,44 & 3,20 & 0,99 & & 3,60 & 2,63 & 2,43 & \\
\hline \multirow{3}{*}{$\begin{array}{c}\text { Septembre } \\
84\end{array}$} & $n$ & 19 & 42 & 31 & 6 & 31 & 50 & 24 & (1) \\
\hline & $\mathrm{m}$ & 41,95 & 47,14 & 50,16 & 51,00 & 41,26 & 51,76 & 53,25 & (65) \\
\hline & $\sigma$ & 1,96 & 2,91 & 1,82 & 4 & 2,26 & 4,21 & 5,58 & \\
\hline \multirow{2}{*}{\multicolumn{10}{|c|}{ R. esculenta }} \\
\hline & & & & & & & & & \\
\hline \multirow{3}{*}{$\begin{array}{c}\text { Septembre } \\
82\end{array}$} & $n$ & 27 & 24 & 6 & 2 & 14 & 7 & (1) & (1) \\
\hline & $\mathrm{m}$ & 59,52 & 69,71 & 70,67 & 73,00 & 60,71 & 71,86 & (79) & (79) \\
\hline & $\sigma$ & 3,72 & 2,89 & 2,43 & 0,00 & 2,81 & 4,12 & & \\
\hline \multicolumn{10}{|l|}{ Site 3} \\
\hline \multirow{3}{*}{$\begin{array}{l}\text { Juillet } \\
\qquad 82\end{array}$} & $\mathrm{n}$ & 4 & 7 & 4 & 1 & 6 & 9 & 8 & \\
\hline & $m$ & 44,50 & 55,57 & 56,00 & (63) & 44,67 & 59,67 & 66,00 & \\
\hline & $\sigma$ & 5,17 & 3,66 & 3,32 & & 3,35 & 5,25 & 4,12 & \\
\hline \multirow{3}{*}{$\begin{array}{c}\text { Septembre } \\
82\end{array}$} & $n$ & 3 & 2 & & & 2 & 3 & (1) & (1) \\
\hline & $\mathrm{m}$ & 54,00 & 56,50 & & & 59,50 & 63,67 & (76) & (70) \\
\hline & $\sigma$ & 7,00 & 4,95 & & & 3,54 & 2,31 & & \\
\hline \multirow{3}{*}{$\begin{array}{c}\text { Septembre } \\
84\end{array}$} & $n$ & 12 & 5 & 1 & & 7 & 8 & 3 & \\
\hline & $\mathrm{m}$ & 45,67 & 56,20 & (63) & & 41,57 & 58,25 & 59,00 & \\
\hline & $\sigma$ & 3,77 & 2,04 & & & 3,11 & 6,16 & 3,27 & \\
\hline \multicolumn{10}{|l|}{ Site 5} \\
\hline \multirow{3}{*}{$\begin{array}{c}\text { Septembre } \\
82\end{array}$} & $n$ & 6 & 10 & 3 & 1 & 11 & 12 & 4 & 1 \\
\hline & $m$ & 54,67 & 62,00 & 65,00 & (64) & 51,55 & 67,00 & 77,50 & (92) \\
\hline & $\sigma$ & 2,13 & 3,71 & 3,77 & & 2,97 & 5,77 & 5,55 & \\
\hline \multirow{3}{*}{$\begin{array}{c}\text { Septembre } \\
84\end{array}$} & $n$ & 9 & 20 & 10 & & 14 & 13 & 2 & \\
\hline & $\mathrm{m}$ & 52,11 & 57,00 & 59,00 & & 54,00 & 59,46 & 66,00 & \\
\hline & $\sigma$ & 6,12 & 4,68 & 3,46 & & 3,98 & 3,93 & 3,00 & \\
\hline
\end{tabular}


Tableau III : Différences de croissance entre les femelles et les mâles de même âge ( $d l$ = différence entre les tailles moyennes ; test $F$ sur les variances ; test $t$ de Student sur les moyennes).

Table III : Differences of frogs growth between females and males of same age. ( $d l=$ difference between mean lengths ; $F$ - test on variances ; $t$ - Student test on means).

\begin{tabular}{|c|c|c|c|c|c|}
\hline & & \multicolumn{4}{|c|}{ Ages } \\
\hline & & $1+$ & $2+$ & $3+$ & $4+$ \\
\hline \multicolumn{6}{|l|}{$\begin{array}{l}\text { R. lessonae } \\
\text { Site } 2\end{array}$} \\
\hline Septembre & $d l$ & 1,00 & 1,74 & 2,33 & 1,93 \\
\hline 85 & $\mathrm{~F}$ & ++ & - & + & - \\
\hline & $t$ & - & ++ & - & - \\
\hline \multicolumn{6}{|l|}{ Site 3} \\
\hline Juillet & dl & $-1,27$ & 7,31 & 3,43 & \\
\hline 82 & $\mathrm{~F}$ & - & - & - & \\
\hline & $\mathrm{t}$ & - & +++ & ++ & \\
\hline Septembre & dl & $-1,62$ & 4,03 & 8,81 & \\
\hline 82 & $F$ & - & - & + & \\
\hline & $\mathrm{t}$ & ++ & +++ & +++ & \\
\hline Septembre & dl & $-0,69$ & 4,62 & 3,09 & \\
\hline 84 & $F$ & - & + & ++ & \\
\hline & $t$ & - & ++ & ++ & \\
\hline \multicolumn{6}{|l|}{ R. esculenta } \\
\hline Septembre & $\mathrm{dl}$ & 1,19 & 2,15 & $(8,3)$ & $(6)$ \\
\hline 82 & $\mathrm{~F}$ & - & - & - & - \\
\hline & $t$ & - & - & $(+)$ & \\
\hline \multicolumn{6}{|l|}{ Site 3} \\
\hline Juillet & $\mathrm{dl}$ & 0,17 & 4,10 & 10,00 & \\
\hline 82 & $\mathrm{~F}$ & - & - & - & \\
\hline & $t$ & - & - & ++ & \\
\hline Septembre & $d l$ & 5,50 & 7,17 & & \\
\hline 82 & $F$ & - & - & & \\
\hline & $t$ & - & - & & \\
\hline Septembre & dl & $-4,10$ & 2,05 & (4) & \\
\hline 84 & $F$ & - & + & - & \\
\hline & $t$ & + & - & - & \\
\hline \multicolumn{6}{|l|}{ Site 5} \\
\hline Septembre & $\mathrm{dl}$ & $-3,12$ & 5,00 & 12,50 & \\
\hline 82 & $F$ & - & - & - & \\
\hline & $t$ & + & + & + & \\
\hline Septembre & $\mathrm{dl}$ & 1,89 & 2,46 & 7,00 & \\
\hline 84 & $F$ & - & - & - & \\
\hline & $\mathrm{t}$ & - & - & + & \\
\hline
\end{tabular}




\subsection{Influence du site}

Ainsi, en septembre 1982, les $R$. esculenta du site 1 présentent une meilleure croissance que celles du site 5, par contre les sites 3 et 5 sont comparables sauf pour les femelles $1+$ (Tab. IV). Ces résultats sont à relier aux caractéristiques propres à chacun des biotopes, en particulier au niveau de leurs richesses trophiques.

Tableau IV : Différences de croissance entre les sites de prélèvement de $\boldsymbol{R}$. esculenta en septembre 1982 (tests = idem Tab. III).

Table IV : Differences of frogs (R. esculenta) growth between sites in september 1982 (Tests $=$ see Tab. III).

\begin{tabular}{|c|c|c|c|c|}
\hline & & & Ages & \\
\hline & & $1+$ & $2+$ & $3+$ \\
\hline \multicolumn{5}{|l|}{ Site 1 comparé au site 5} \\
\hline & $\mathrm{dl}$ & 4,85 & 7,71 & 5,67 \\
\hline mâles & $F$ & - & - & - \\
\hline & $\mathrm{t}$ & ++ & ++ & ++ \\
\hline & $\mathrm{dl}$ & 9,16 & 4,86 & \\
\hline femelles & $F$ & - & - & \\
\hline & $t$ & ++ & +++ & \\
\hline \multicolumn{5}{|l|}{ Site 5 comparé au site 3} \\
\hline & $\mathrm{dl}$ & 0,67 & 5,50 & \\
\hline mâles & $F$ & - & - & \\
\hline & $\mathrm{t}$ & - & - & \\
\hline & $d l$ & $-7,95$ & 3,33 & \\
\hline femelles & $F$ & - & - & \\
\hline & $t$ & ++ & - & \\
\hline
\end{tabular}

\section{4. Évolution estivale}

La comparaison des tailles moyennes de juillet à septembre permet de suivre la reprise de croissance après la période de reproduction (Tab. V). L'exemple du site 3 en 1982 montre une croissance significative pour $R$. lessonae au niveau des $1+$, des $2+$ sur les deux sexes, des $3+$ sur les femelles. En revanche pour $R$. esculenta, seule la croissance des $1+$ est significative.

\section{5. Évolution interannuelle}

Il est possible de comparer les tailles moyennes en septembre 1982 à celles de septembre 1984 pour les sites 3 et 5 (Tab. VI). Sur le site 3 la taille des $R$. lessonae est significativement plus élevée en 1982 pour tous les âges et les deux sexes, elle l'est seulement au niveau des $1+$ pour $R$. esculenta. La tendance est la même pour le site 5 pour la plupart des âges chez R. esculenta. 
Bull. Fr. Pêche Piscic. (1991) $321: 55-71 \quad-69-$

Tableau V : Différences de croissance entre juillet et septembre 1982 pour le site 3 (tests = idem Tab. III).

Table V : Differences of frogs growth between July and September 1982 in site 3 (Tests = see Tab. III).

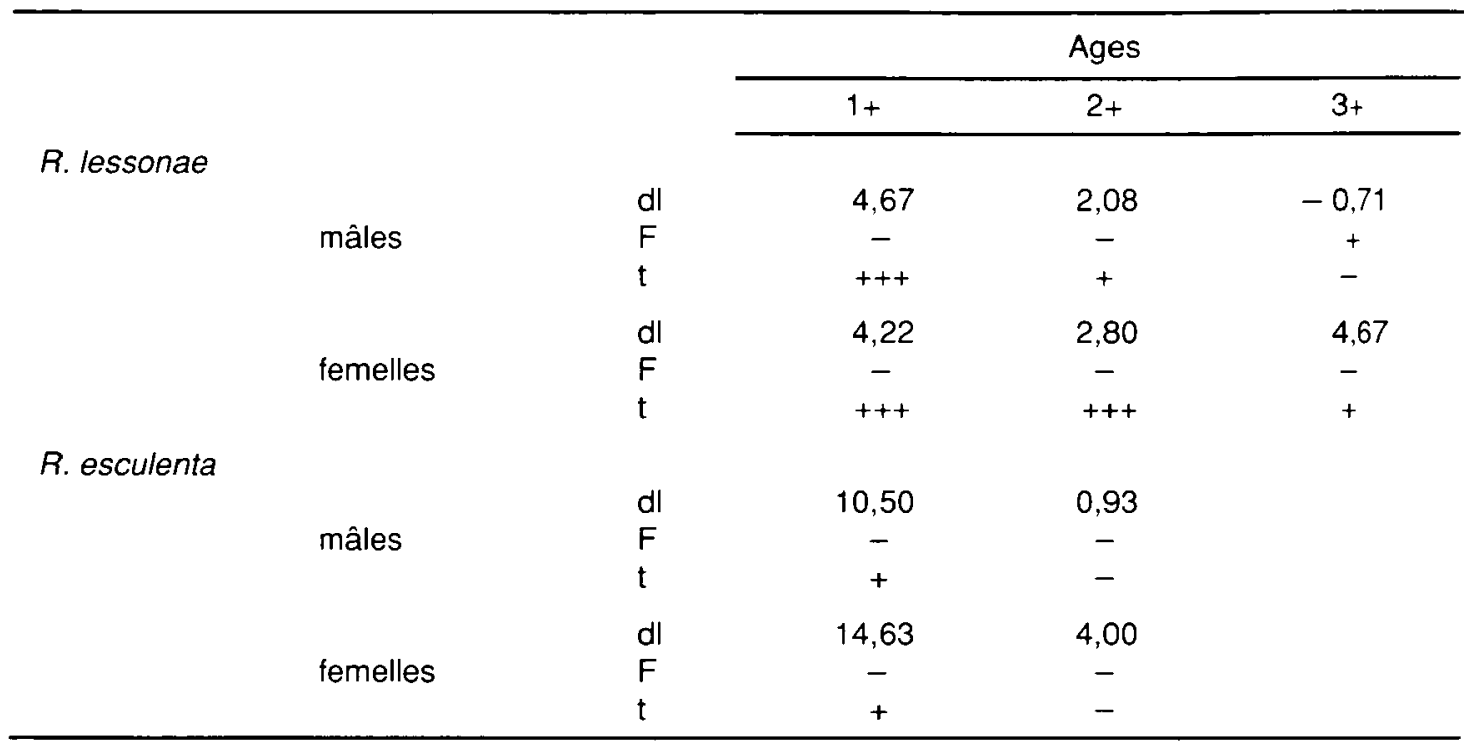

Tableau VI : Différences de croissance entre les années 1982 et 1984 (tests = idem Tab. III).

Tableau VI : Differences of frogs growth between two years 1982 and 1984 (Tests = see Tab. III).

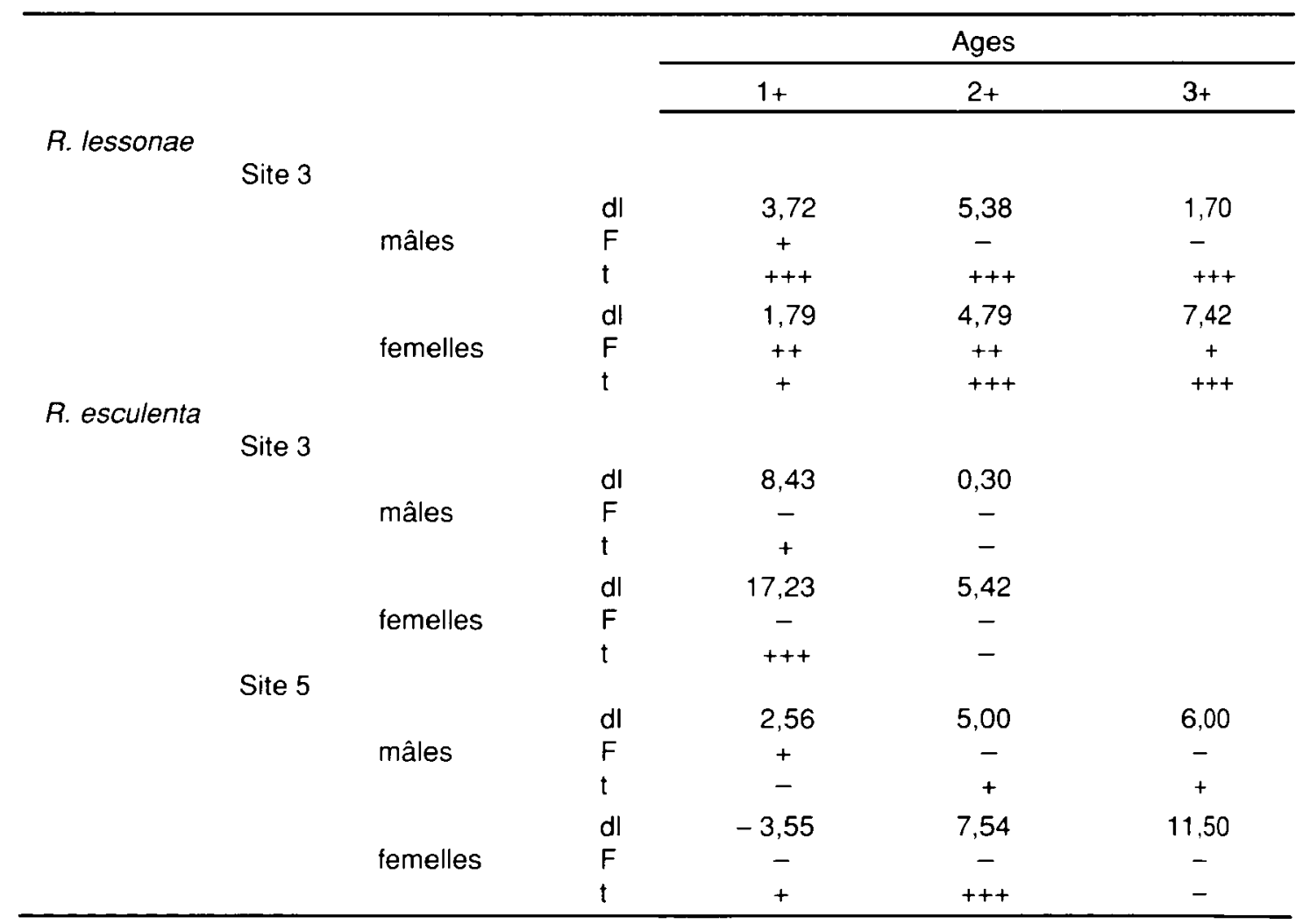




\section{DISCUSSION - CONCLUSION}

Les échantillons examinés ont été diversifiés dans le temps et l'espace afin de définir un aspect moyen régional. Ils ne permettent pas un suivi régulier de l'influence des divers facteurs, en particulier d'une année à l'autre. Ce genre d'étude est aussi limité par la nécessité de ne pas abattre un trop grand nombre d'animaux dans chaque site et par la lourdeur des études squeletto-chronologiques.

Les résultats montrent que, dans un but d'homogénéité, il est préférable d'analyser les structures démographiques en septembre juste avant l'hivernage et lorsque les caractères sexuels secondaires externes des individus $1+$ sont visibles. A cette époque les populations sont globalement représentées par deux groupes de tailles : des subadultes de l'année précédente et des adultes constitués de plusieurs âges de tailles semblables.

La détermination de l'âge à partir des coupes de tibias permet de répartir les adultes en $2+, 3+$ et quelques rares individus plus âgés. Cette structure est la même chez $R$. lessonae et $R$. esculenta, ainsi que chez les deux sexes. La part relative des animaux de 1 an est très variable en liaison avec une capturabilité plus faible liée à une taille plus petite, mais aussi à une localisation plus terrestre au niveau des berges. Les rares données concernant l'âge de grenouilles vertes sont celles de SHALDYBIN (1976) et de BORKIN et al. (1979) qui trouvent des animaux plus âgés (jusqu'à 12 ans) pour l'URSS en liaison avec un climat plus rude. Les histogrammes des tailles de BLANKENHORN (1974), obtenus en Suisse, sont plus proches.

Le taux de mortalité est peu élevé entre le $1^{\text {er }}$ et le $2^{e}$ été, il augmente ensuite. II n'est pas rare de trouver des animaux morts après la ponte et à l'entrée de l'hivernage. A cette époque les adultes semblent plus sensibles au syndrome septicémique. Les plus grands individus, mais aussi les plus rares, sont généralement des femelles. Les mortalités affectent autant les deux sexes, c'est aussi ce que trouve SHALDYBIN (1976) et BORKIN et al. (1979), mais ce n'est pas le cas du crapaud commun (GITTINS, 1983).

La croissance est plus lente chez $R$. lessonae en rapport avec une taille finale plus faible. De même la maturité sexuelle plus précoce chez la plupart des mâles, qui se traduit par l'apparition des caractères sexuels externes dès la première année, entraîne un ralentissement de la croissance et une différence de taille en faveur des femelles à partir de 2 ans. En revanche il est plus difficile d'expliquer la plus grande taille des mâles à 1 an, fait signalé par BERGER (1970) en Pologne sur des populations vivant en parcs extérieurs. Si les tailles moyennes obtenues par les auteurs soviétiques sont plus faibles, celle de BLANKENHORN (1974) pour la Suisse sont un peu plus élevées qu'en Bretagne.

En fait, certains milieux sont plus favorables à la croissance en particulier les étangs et certaines mares en zone agricole (pour $R$. esculenta). L'eutrophisation, les déjections de bovins doivent favoriser la production de proies (invertébrés divers, Diptères). Mais il faut remarquer que les densités de populations sont aussi plus faibles dans ces biotopes comparativement aux marais à $R$. lessonae (données non publiées).

Il est donc difficile de savoir lequel des deux facteurs influe le plus : abondance de la nourriture ou compétition interindividus.

La climatologie de l'année semble aussi agir sur la croissance par l'intermédiaire des ressources trophiques et du niveau d'activité.

Cette première approche des structures démographiques montre les difficultés d'interprétation de l'évolution des populations sans un suivi régulier et une analyse précise de la croissance. Dans ce but, la lecture de l'âge individuel sur les os doit être améliorée par la recherche d'un maximum d'informations sur l'histoire de l'individu associée à une standardisation des rétromesures. Quant aux différences de croissance suivant les sites, il reste à démontrer l'importance relative des facteurs trophiques et densité dépendants.

\section{BIBLIOGRAPHIE}

BERGER L., 1970. Some characteristics of the crosses within Rana esculenta complex in postlarval development. Ann. Zool., 27, 373-416. 
BLANKENHORN H.J., 1974. Soziale Organisation einer Mischpopulation Von Rana lessonae Camerano und Rana esculenta L. Unpubl. Dissertation, Univ. Zürich, $100 \mathrm{p}$.

BORKIN L.J.A., GARANIN W.I., TICHENKO N.T., ZAUNE I.A., 1979. Some results in the green frogs survey in the USSR. Mitt. Zool. Mus. Berlin, 55, 153-170.

FRANCILLON H., CASTANET J., 1985. Mise en évidence expérimentale du caractère annuel des lignes d'arrêt de croissance squelettique chez Rana esculenta $L$. (Amphibia, Anura). C.R. Acad. Sci. Paris, 300, 327-332.

GIBBONS M., Mc CARTHY T.K., 1983. Age determination of frogs and toads (Amphibia, Anura) from North Western Europe. Zool. Scr., 12, 145-151.

GITTINS S.P., 1983. Populations dynamics of the common toad (Bufo bufo) at a lake in Mid-Wales. J. Anim. Ecol., 52, 981-988.

GITTINS S.P., STEEDS J., WILLIAMS R., 1982. Population age structure of the common toad (Bufo bufo) at a lake in Mid Wales determined from annual growth rings in the phalanges. Br. J. Herpetol., 6, 249-252.

GUYETANT R., CASTANET J., PINSTON H., 1984. Determination de l'âge de jeunes grenouilles Rana temporaria L. par l'analyse des marques de croissance de coupes transversales d'os compact. C.R. Soc. Biol., 178, 271-277.

GUYETANT R., BROSSE S., HEROLD J.P., PINSTON H., 1988. Étude de la croissance et du développement de grenouilles rousses Rana temporaria L. en altitude (Alpes du Nord). C.R. Soc. Biol., 182, 301-307.

HEMELAAR A.S.M., VAN GELDER J.J., 1980. Annual growth rings in phalanges of Bufo bufo (Anura, Amphibia) from the Netherlands and their use for age determination. Nether. J. Zool., 30, 129-135.

REGNIER V., 1983. Étude écologique des grenouilles du complexe Rana esculenta L. en Pays de Vilaine (Bretagne). Thèse Doct. 3 e cycle, Univ. Rennes, $115 \mathrm{p}$.

REGNIER V., NEVEU A., 1986. Structures spécifiques des peuplements en grenouilles du complexe Rana esculenta de divers milieux de l'Ouest de la France. Acta Oecologica, Oecol. Applic., 7, 3-26.

RYSER J., 1988. Determination of growth and maturation in the common frog, Rana temporaria, by skeletochronology. J. Zool., Lond., 216, 673-685.

SCHERER B., 1984. Biostatistique. G. Morin Ed., Boucherille, Canada, 847 p.

SHALDYBIN I.P., 1976 in BORKIN et al., 1979. 